\title{
The Most Simple and Convenient Synthesis of the Baylis-Hillman Adducts of Cycloalkenones: Use of DMAP in Aqueous THF
}

\author{
Ka Young Lee, Ji Hyeon Gong, and Jae Nyoung Kim* \\ Department of Chemistry and hrstitute of Basic Science, Chonnam Nationat Universin: Kwangit 500-757, Korea \\ Recened February 23, 2002
}

Keywords : Baylis-Hillman reaction, DMAP, Cycloalkenones, Baylis-I lillman adducts.

The Baylis-][illman reaction, the coupling of aldehydes and activated vinyl compounds, is one of the most important carbon-carbon bond-foming reaction in organic synthesis. The drawback to this methodology is its slow reaction rate, and many research groups have examined a varicty of methods to accelerate the reaction. Among the activated alkenes cycloalkenones are lamous. ${ }^{\text {tb }}$ Under the nomal reaction conditions using DABCO (1.4-diazabicyclo[2.2.2]octane) no reaction was observed.

Recently, various methods for the synthesis of the Baylislillman adducts of eycloalkenones have been developed with certain limitations. (1) The combination of 2.6-diphenyl-4H-chalcogenopyran-4-ones (or -4-thions) and $\mathrm{TiCl}_{+}$in methylene chloride ${ }^{2 i}$ and related methods were developed for the reactive $p$-nitrobenzaldehyde and 2-cyclohexen-1one or 2-cyclopenten-1-onc. ${ }^{2}$ (2) The combination of lithium perchlorate $\left(\mathrm{LiClO}_{4}\right)$ and $\mathrm{D} \triangle \mathrm{BCO}$ in ether could be used lor the Baylis-f Iillman reaction of benzaldehyde and 2-cyclohexen-1-one." (3) The use of $\mathrm{El}_{2} \mathrm{AlI}$ in $\mathrm{CJ}_{2} \mathrm{Cl}_{2}$ for the reaction of enone isolevoglucosenone and $C$ - $\beta$-D-galactopyranosylformaldehyde furnished the cortesponding Baylisl Lillman adduct. ${ }^{+}$(4) 4-(Dimethylamino)pyridine (DMAP)catalyzed hydroxymelhylation of 2-cyclohexenones with lormaldchyde was carried out eflectively in aqueous medium." (5) Tributylphosphine combined with 1, l'-bi-2-naphthol was used effectively for cycloalkenone system. (6) Lewis base effects including DMAP, tributylphosphine and DBU (1.8diazabicyclo[5.4.0]undec-7-ene) in the Baylis-Hillman reaction of 2-cyclohexen-1-one and arylaldehyde $N$-tosylimines were examined. ${ }^{7}$ (7) Recently, it was known that $\mathrm{TiCl}_{4}$ without the use of lewis base could be applied to highly reactive aldehydes. ${ }^{8}$ (8) lithium phenylselenide (PhSel.i) induced Baylis-Hillman reaction was applied successively to $\alpha, \beta$-unsaturated lactone system. ${ }^{3}$ In summary, for the BaylisHillman reaction of cycloalkenone derivatives, the combination of $\mathrm{L}$ ewis acid and lewis base system ${ }^{2.3 .6}$ seemed the only choice except for the reactive formaldehyde. However, most of the reported methods was limited to reactive aldehydes with low to moderate yields of products.

In the course of our program for the synthesis of quinoline derivatives via $\mathrm{S}_{2} 2^{\prime}-\mathrm{S}$ Ar-elimination strategy. ${ }^{(1)}$ we would like to extend the scope to the cycloalkenone derivatives. Thus, we needed the Baylis-Hilman adducts of 2-cyclohexen-1-one and various arylaldehydes. The use of DMAP, DBU. PBus or DABCO as a base catalyst did not give the desired products in TH IF or $\mathrm{CI}_{2} \mathrm{Cl}_{2}$. The use of expensive 2cyclohexen-1-one as solvent is nol clever. The use of known acid-base combination methods is not conveniently accessible to us. Thus, we intended to search for the simple conditions for the reaction. Among the examined conditions, the use of DMAP (0.2 equiv) in aqueous THIF (1:1) suffice the formation of the desired Baylis-][illman adducts in reasonable yiclds." $\mathrm{As}$ shown in Scheme 1 and in Table 1 , we examined 2-cyclohexen-1-one (2a) and 2-cyclopenten-1one (2b) and report herein the results.

The reaction is simple and straightforward. '? $A$ mixture of aldehyde 1, cycloalkenone 2 and DMAP (0.2 equiv) in aqueous TIJF (1:1) was stirred at room temperature for the time given in Table 1 . Alter the usual workup process and column chromatographic purilication (hexanciether, 6:4). we could obtain the Baylis-I Jillman adducts 3 and the results are summarized in Table $1 .^{12}$ During the course of this work. Aggarwal et al. reported on the rate acceleration of the Baylis-IJillman reaction in polar solvents such as water and fomamide. ${ }^{13}$ They examined various amines including DABCO, DM $\wedge$ P, 3-hydroxyquinuclidine (3-I IDQ) and found that the use of 3-JIDQ showed best results. Ilowever, the reaction used expensive $3-$ IDQ in equimolar amounts. In view of yields and the use of easily available DMAP in catalytic amounts, our conditions seemed more general and convenient than the $\Lambda$ ggarwal's method.

The reaction mechanism is as follows as shown in Scheme 1. A conjugated addition of a Lewis base (DMAP) to

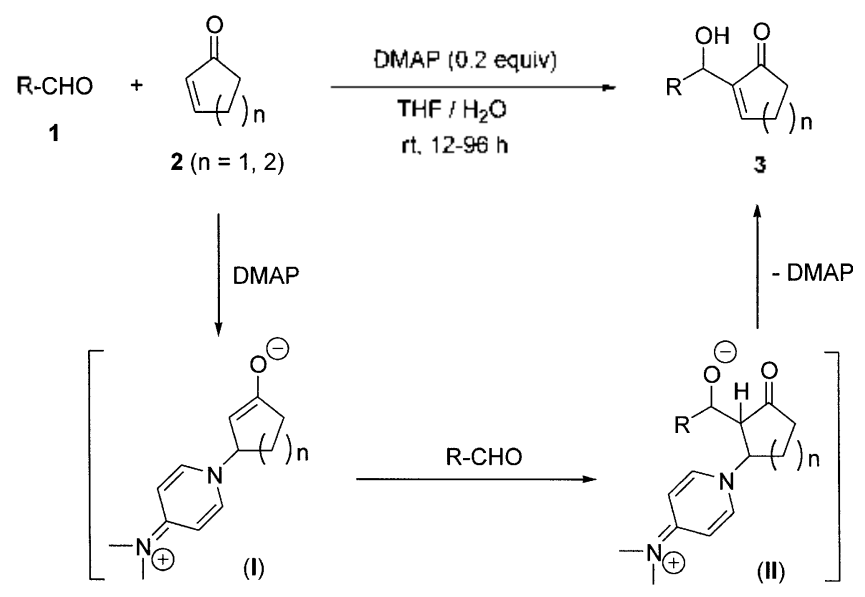

Scheme I 
Table 1. Synuhesis of the Baylis-Hillman adducts of 2 -cycloalkenI-ones

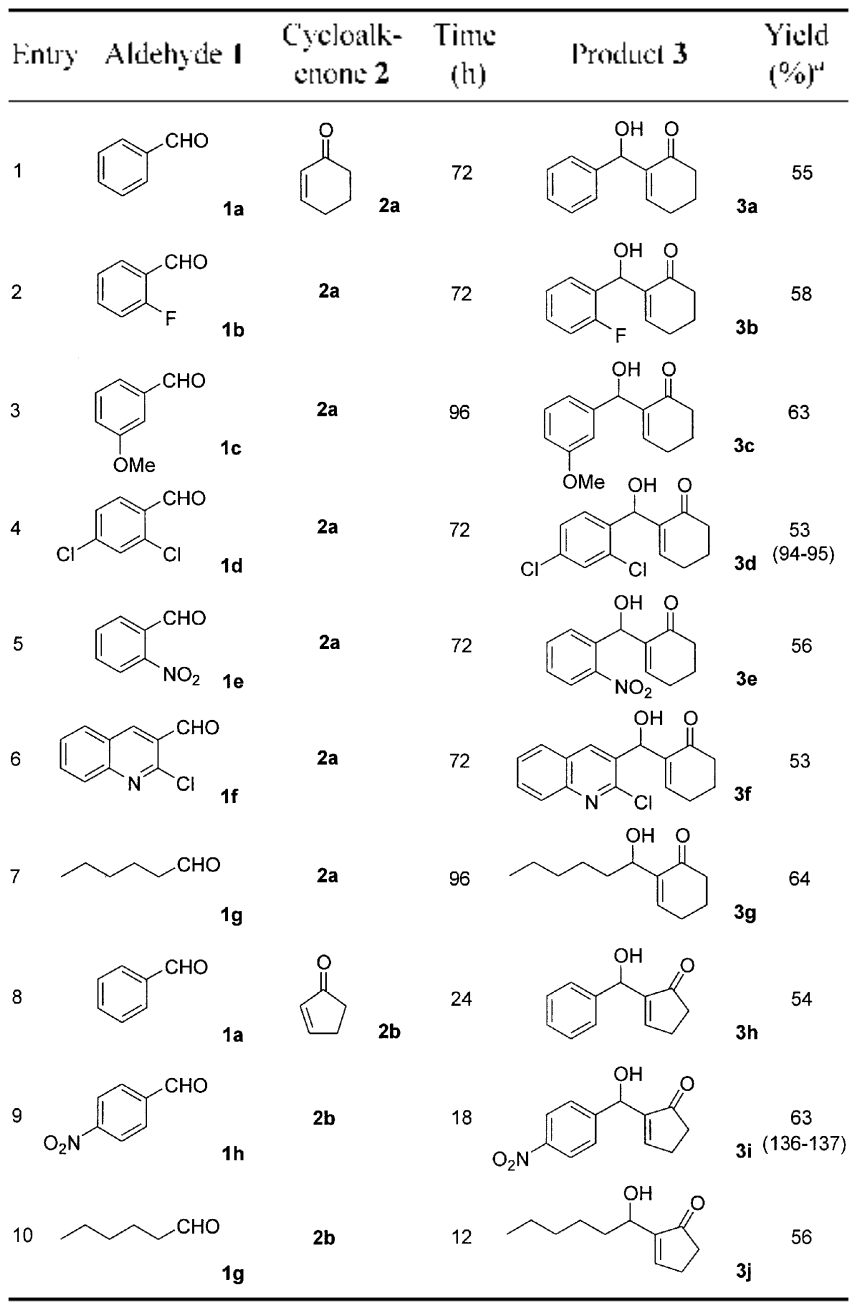

" $\mathrm{Mp}$ was wfitten in parenthesis for $\mathbf{3 d}$ and $3 \mathbf{i}$ and others are oil.

cycloalkenone 2 affords an enolate (I), which reacts with an aldehyde to give an aldol-type intermediate (II) followed by $\beta$-elimination of DMAP' to give the product 3 . As shown the reaction sequence involves charged transition states and intermediates that could be stabilized by polar solvents such as water through intermolecular charge-dipole interactions as well as hydrogen bonding interactions to accelerate the reaction rate. ${ }^{1.6 .1 .1 .14}$

As a conclusion we disclosed a simple and convenient reaction conditions for the Baylis-Hillman adducts of cycloalkenones.

Acknowledgment. This work was supported by a Korea Research Foundation Grant (KRF-2001-015-DP0326). The support of the Korea Basic Science Institute (Kwangju Branch) is also acknowledged.

\section{References and Notes}

1. (a) Basavaiah. D.: Rao. P. D.: Iyma. R. S. Tetrohedron 1996. 52. 8001. (b) Ciganek, I: Organic Reactions; Ioln Wiley \& Sons: New York. 1997: Vol. 5l. pp 201-350. (c) Drewes. S. E.: Roos. (j. H. P. Tetrahedron 1988. H4. 4653. (d) Langer. P. Angew. Chem., im. Ed. Engl. 2000. 39. 3049.

2. (a) Jwama. T.: Kinoshita. I.: Kataoka. T. Tetwhedron Leff. 1999. 40. 3741. (b) Kataoka. T.: Iwama. T.: Tsujisama S.-i. Chen.

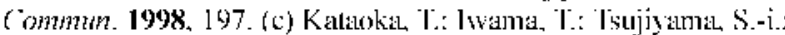
Iwamura. 'L: Watanabe. S.-i. Fetrahedron 1998. 5f. 11813. (d) Bauer. J.: larasiuk. J. Tetrthedron: Aswnmetry 2001. 12. 1741.

3. Kawamura. M.: Kobayashi. S. Tetrehedron Lett. 1999. 40.1539.

4. 7.hu. Y.-I1.: Vogel. P. Symlet 2(0)1. 79.

5. (a) Rezgui, F., F:I Gaied. M. M. Tetrahedron Iett, 1998. 39. 5965. (b) Sugahara T:: Ogasawarth K. Sinlet 1999. 419.

6. Yamada Y. M. A.: Ikegani. S. Tetrahedron Leth 2000. H.2165.

7. Shi. M.: Xu. Y.-M. J. Chem. Soc. Chem. Commen. 2001. 1876.

8. Li. G.: Wei. H.-X.: Gao. J. J.: Caputo. T. D. Tetrohedron Lett. 2000. $H$. I.

9. Jauch. J. J. Org (hem. 2001.66.609,

10. (a) Kim. J. N.: I.ee. II. J.: I.ee. K. Y.: Kim. II. S. Fetrathedron lett. 2001. 42. 3737. (b) Chung. Y. M.: Lee. H. I.: Hwang. S. S.: Kirn. J. N. Bull. Komean (hem. Soc. 2001. 22.799.

11. When we used DABCO (0.2 equiv) as a base catalyst in THF or C $\mathrm{HI}_{2} \mathrm{Cl}_{2}$ desired Baylis-Hillman adduct was not synthesized in appreciable amounts ${ }^{\text {ia }}$ When the reaction was performed without additional solvent (cyclohexen-1-one acts as at reagent and as a solvent in this case) product was oblained in low yield. However. this is not clever as mentioned thefore. The use of $\mathrm{DABCO}$ in aqueous THF showed intractable products. $\mathrm{DBL}$ or $\mathrm{PBu}_{4}$ in agucous THF showed complex mixtures. too. The use of DMAP in dry THF or in moisturized THF showed diminished vields of producls.

12. To a stirred solution of aldehyde I ( 2 mmol) in aqueous $\mathrm{THF}$ (3 mL. $\left.\mathrm{H}_{2} \mathrm{O} / \mathrm{lHH} .1: 1\right)$ was added cycloalkenone $2(2 \mathrm{mmol})$ and DVAP (49 mg. $0.4 \mathrm{mmol}$ ) at room temperature. After being stirred for the time given in Table I, the desired Baylis-Ilillman adducts $3 \mathrm{a}$-j were obtained by normal workup process and column chromattography (hexaneiether. $3: 1$ ). Spectroscopic datti of 3a and 3h is as follows. 3a ${ }^{2 \pi}$ : clear oil: $\operatorname{IR}(\mathrm{KBr}) 3421.1669 \mathrm{~cm}^{-1}$ : 'H V.MR (CDCls) $\delta 1.92-2.04$ (m. 2H). 2.32-2.48 (m. $4 \mathrm{H}$ ). 3.10 (brs. 1H). 5.55 (s. $1 \mathrm{H}) .6 .74$ (t. $J=4.2 \mathrm{~Hz} .1 \mathrm{H}) .7 .22-7.38$ (m. $5 \mathrm{H}$ ): ${ }^{1.3} \mathrm{C}$ NMR (CDCl $) \delta 22.51,25,75,38.56,72,51,126.44,127,45$. 128.28. 141.10, 141.69, 147.25,200.31: Mass $(70 \mathrm{eV}) m=$ (rel. intensity) 77 (23). 105 (14). $128(23) .201$ (100). 202 (M'. 47). $3 h^{\prime \prime}$ : clear oil: IR (KBr) 3422. $1686 \mathrm{~cm}^{-1}$ : 'H NMR (CLCl $\delta$ $2.40-2.45$ (n. $2 \mathrm{H}$ ). $2.54-2.60$ (n). $2 \mathrm{H}$ ). 3.59 (d. J $3.9 \mathrm{~Hz} .1 \mathrm{H}$ ). $5.54(\mathrm{~s}, 1 \mathrm{H}) .7 .25-7.40(\mathrm{~m} .6 \mathrm{H}):{ }^{1.4} \mathrm{C}$ NMR $(\mathrm{CDCl}) \delta 26.57$.

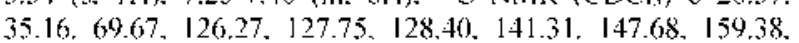
209.54: Mass (70 eV) $m=$ (rel, intensity) 77 (30), 105 (I8). 115 (17). $128(100)$. $145(18) .187$ (47). $188\left(\mathrm{M}^{\prime} .72\right)$.

13. Agganval. V. K.: Deans. D. K.: Mereu. A.: Willians. R. $J$. Org Chem. 2002. 67. 510 .

14. Yu. C.: Liu. B.: Hu. L. J. Oig. Chem. 2001. 66. 5413. 